\title{
ROBUST BUILDING ROOF SEGMENTATION IN AIRBORNE POINT CLOUD DATA
}

\author{
Ali Naqi Gilani ${ }^{1, \star} \quad$ Mohammad Awrangjeb ${ }^{2,} \quad$ Guojun Lu $^{3,}$ \\ ${ }^{1}$ Faculty of Information Technology, Monash University, Australia \\ ${ }^{2}$ School of Info. and Comm. Tech., Griffith Sciences, Griffith University, Australia \\ ${ }^{3}$ School of Eng. and Info. Tech., Federation University Australia, Australia
}

\begin{abstract}
Approximation of the geometric features is an essential step in point clouds segmentation and surface reconstruction. Often, the saliency features are estimated using principal component analysis (PCA), which is sensitive to noise and tend to smooth the sharp features. Hence, the segmentation results into unreliable reconstructed surfaces. This article presents a new approach to segment the point clouds in order to reconstruct roofs and detect buildings. It combines PCA and Low-Rank Subspace with prior Knowledge (LRSCPK) methods for normal estimation. The points around the anisotropic surfaces are identified using local neighbourhood covariance analysis followed by the normal estimation using PCA. Then, the normals for the points around sharp features are re-estimated using a consistent isotropic sub neighbourhood by LRSCPK. Demonstration on two real-world ALS (Airborne Laser Scanning) samples shows the ability of the combined approach to generate more accurate and robust normals. The developed segmentation approach is further tested using two benchmark datasets: ISPRS and Australian. Per-object and per-area completeness and correctness results indicate the robustness of the approach and the quality of the reconstructed surfaces and extracted buildings.
\end{abstract}

Index Terms - Segmentation, Detection, Reconstruction

\section{INTRODUCTION}

The acquisition of precise 3D spatial data at real-time over a large area has become possible with the advancement of modern sensing devices. Over time, remote sensing data has become the most important resource to capture 3D complex models, because of reliability, a degree of automation and speed characteristics. That is why, its use in widespread for various applications in surface reconstruction, cadastral and disaster management, city planning, and damage assessment [1].

Segmentation is an important intermediate task which separates and labels the most similar surface points into homogeneous surfaces. Generally, the building detection process is aimed at identifying planar segments from point clouds as most elements of the buildings are planar surfaces. However, an accurate segmentation of individual elements of the buildings is not trivial due to various reasons, such as 1) the 3D points carries no connection information and unorganised, 2) usually sparse and some time has gaps, which results in a single planar surface to fragment into several small segments, or lead to inaccurate

\footnotetext{
*Corresponding author: alinaqi.gilani@monash.edu
}

segmentation; 3) presence of noise points due to physical limitation of ALS sensors, occlusion, or multiple reflectance; 4) the surface shape can have sharp features with no statistical distributional pattern, and 5) point clouds from non-planar surfaces or irregular objects are not recognised by any plane segmentation methods.

In surface reconstruction, the quality of the extracted surface heavily depends on the quality of the estimated normal. Consequently, the high-quality normals can significantly improve the performance of a segmentation process even in the presence of noise [2]. Numerous algorithms rely on the quality of normal estimation in point clouds, such as point-based rendering, surface reconstruction, 3D piecewise-planar reconstruction, and primitive extraction [3]. PCA is being used commonly for normal and curvature estimation using a fixed local neighbourhood [4]. There exists several variation of it, but all these techniques tend to smooth the sharp features mainly due to PCA's non-robust location and scatter analysis. Thus, erroneous saliency features (normal and curvature) result in unreliable and inaccurate surface extraction. Mitra and Nguyen [5] also discuss the sensitivity of PCA to noise in plane fitting to estimate point normal. To overcome this limitation, several methods [2, 3, 6] are proposed which provide robust normals along the sharp features.

This paper proposes a statistically robust building roof segmentation technique. We first extract the non-ground point clouds and estimate a normal on each point cloud using PCA, whereas normals estimated on sharp features are refined using LRSCPK [2]. The rest of the paper is organised as follows. Section 2 briefly discusses the relevant normal estimation methods and principles. Section 3 narrates the robust building feature extraction technique using a test dataset. Section 4 provides performance evaluation and comparative analysis of the proposed approach using two benchmark datasets. Finally, the proposed research is concluded in Section 5.

\section{RELATED WORK AND PRINCIPLES}

According to Chen et al. [7], building detection and reconstruction approaches have two basic categories: model-driven and data-driven. The model-driven methods begin with a selection of a hypothetical model and then verify the model using ALS point clouds. These methods are limited to the buildings which have their models present in the list. The extracted roofs using such methods are topologically correct [7]. On the other hand, the data-driven methods are not restricted to some particular shapes and extract the building primitives (roofs, chimneys etc) based on some similarity analysis. These kinds of methods describe 
the detail of the buildings and extend to the wider class of polyhedral models. The success of these methods depends widely on a segmentation method which estimates the building primitives. The proposed method falls into this category.

For a given $3 \mathrm{D}$ point cloud, how accurate the estimated point normal approximates the true normal is indispensable to the success of a segmentation method. These normal estimation methods are classified into two distinct groups: numerical optimisation and computational geometry methods [8]. For the prior category, plane fitting methods [4] are most popular to estimate point normal which approximate a tangent plane with a regression by PCA using a fixed local neighbourhood. Other surfaces have also been used to estimate point normal for example spheres [9]. However, regression-based methods including PCA and its variants $[5,9]$ tend to smooth sharp features, and thus, fail to estimate a correct normal near edges and anisotropic surfaces as shown in Figure 1(a) and 1(c).

To overcome the limitations, Fleishman et al. [6] classifies the point clouds around sharp features into groups. The estimated normals are more accurate and statistically robust. More recent works $[2,3,10]$ address the effect of noise and anisotropic surfaces for normal estimation. Li and Schnabel [10] combine a robust local noise estimator and a kernel density estimation to handle noise and sharp features. But it fails to address point variation on the edges. Boulch and Marlet [3] present a method based on the robust version of the Randomised Hough Transforms. It uses a uniform sampling strategy and a stop criterion to handle noise and density variation on edges. However, it does not work very well with large noise and shallow angles [2, 8]. Computationally geometry methods rely on the construction of Voronoï diagrams. The original Voronoï based algorithm search the farthest vertex of the Voronoï cell to estimate the normal. But it works well in noise free 3D points and hardly handle noise.

\subsection{Principal Component Analysis and LRSCPK}

PCA is a statistical procedure concerned with reducing the dimensionality of the data and identifying new meaningful underlying variables called principal components (PCs). The PCs describe the data variances in different orthogonal directions given by the eigenvectors of the covariance matrix $M$. PCA works as a basis transformation to diagonalise an estimate of the covariance matrix:

$$
M_{3 \times 3}=\frac{1}{N} \sum_{i=1}^{N}\left(p_{i}-\mu_{p}\right)\left(p_{i}-\mu_{p}\right)^{T}
$$

where $p_{i}=\left(x_{i}, y_{i}, z_{i}\right)$ is a $3 \mathrm{D}$ point in ALS point clouds $P$, and $\mu_{p}$ is the centre of $N$ points. The transformation requires to solve the eigenvalue equation $\lambda V=C V$ using Singular Value Decomposition (SVD) on $M$. The eigenvalues $\left(\lambda_{2} \geq \lambda_{1} \geq \lambda_{0}\right)$ and eigenvectors $V\left(v_{2}, v_{1}\right.$ and $\left.v_{0}\right)$ are obtained where each $\lambda_{i}$ describes the spatial variation corresponding to their $v_{i}$.

LRSCPK employs an affinity matrix which is dense in the same class and sparse between different classes in order to segment the points on anisotropic surfaces. The following equation and the model information are adopted from the method in [2].

$$
\min \|Z\|_{*}+\beta\left\|\mathcal{P}_{\Omega}(Z)\right\|_{1}+\gamma\|E\|_{2,1} \quad \text { s.t. } \quad X=X Z+E \text { (2) }
$$

where each column of matrix $X$ represents a 3D point, $\|\cdot\|_{*}$ shows the sum of singular value, $E$ is a soft convergence constraint $\beta$ and $\gamma$ are the constants and $\Omega$ is a guiding matrix having estimated normals as a prior knowledge. The authors showed through experiments that the subspace can be clustered correctly even when $\Omega$ contains a gentle noise. For better understanding, the reader is encouraged to consult the very article.

\subsection{Saliency features and proximity criteria}

Point cloud segmentation procedures often encounter a situation when two or more roof surfaces intersect each other (making shallow or thick angles) or the roof primitives mutually superpose (forming jump edges). Thereby, PCA-based segmentation processes often wrongly approximate surfaces and merge the coincident planes due to smooth normals at sharp features. Besides the noise, outlier, and anisotropic surfaces, the approximation of a point normal also depends on appropriate selection of a local neighbourhood size $N_{p}$ and methods to find a point's $p_{i}$ neighbours. Three commonly used neighbourhood selection methods are k-nearest neighbours (KNN), Delaunay triangulation, and fixed-distance neighbours. KNN supersedes the counterparts since it adapts the data sparsity and thus better suits in airborne mapping data. Therefore, we choose KNN based KD-tree search algorithm to determine a point's local neighbourhood.

According to Hoffman et al. [11], a smaller $N_{p}$ estimates a point normal which is more susceptible to noise, albeit the local geometry is better represented. Many authors suggest to use a larger $N_{p}$ for a better approximation of a normal to cater noise $[2,3,11,12]$, which adversely affect the local characteristics of the normal vector. So, after an empirical study with variable neighbourhood sizes, we choose $N_{p}=20$ for all the experiments so that the neighbours in the local vicinity of a point become coplanar. To demonstrate that PCA alone generates inaccurate normals in the case of anisotropic surfaces, we take small samples from real data to demonstrate the robustness of combined normal estimation approach as shown in Figures 1.

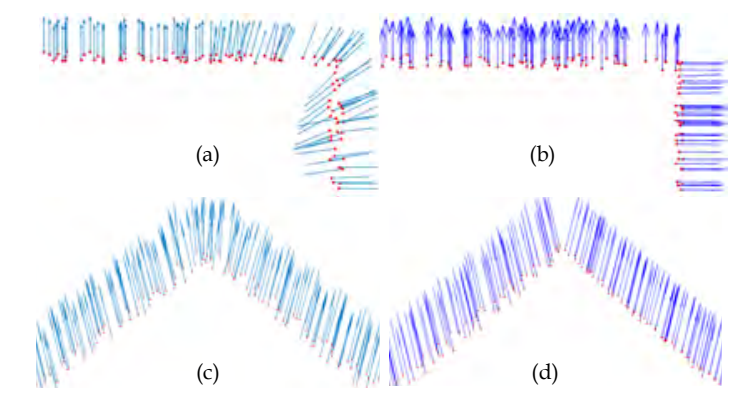

Fig. 1. Reconstructed normals on orthogonal and shallow angled planes - (a, c) PCA, and (b,d) PCA followed by LRSCPK

Generally, the classical region-growing technique augments the neighbouring points using the similarity features (flatness, orientation) to obtain the coplanar surfaces. Since, each PC describes the data variance and particularly in 3D space, a planar surface can be well explained by first two PCs. Therefore, $v_{0}$ approximates the surface normal $\hat{n}$ for $p_{i}$ and $\lambda_{0}$ describes the spatial variation along the surface normal. Similarly, the surface flatness can be measured as $F=1-\lambda_{0} /\left(\lambda_{2}+\lambda_{1}+\lambda_{0}\right)$ [12]. Based on an accurate approximation of normal vector and flatness, the approximated planar surfaces can well describe the true primitives. 


\section{PROPOSED METHODOLOGY}

The workflow of the proposed approach consists of three major steps as shown in Figure 2. Firstly, the candidate building regions are identified after separating the ALS data into a ground and non-ground point clouds. Secondly, the building roof primitives are extracted from each candidate region after point cloud segmentation. Finally, the planes on non-building structures are eliminated using the refinement process followed by the roof plane and building outline approximation. A detailed explanation is provided in the following sections.

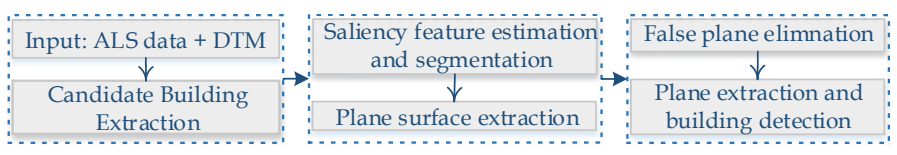

Fig. 2. Workflow of the proposed approach

\subsection{Candidate building region identification}

The proposed approach takes ALS data and a digital terrain model (DTM) with $1 \mathrm{~m}$ resolution as an input. An ALS usually contains point clouds returned from different features such as ground, trees, buildings and other 3D objects. The first step for building roof segmentation is to separate the area of interest from the rest. We, therefore, use a $1 \mathrm{~m}$ height threshold using a ground height from DTM for extraction of these points. This process eliminates low height features including bare-earth, roadside furniture, cars, and bushes while leaving objects above the threshold including buildings and trees.

Out of several methods to select anisotropic points, we use covariance analysis of the neighbourhood of a point [13]. The weight $w_{i}$ is computed as $\lambda_{0} /\left(\lambda_{2}+\lambda_{1}+\lambda_{0}\right)$ which measures the confidence of $p_{i}$ belonging to an edge or corner. Usually, the points around the sharp features have a larger $w_{i}$. If $w_{i}$ is larger than a user-defined threshold then $p_{i}$ is regarded as a feature point as shown in Figure 3.

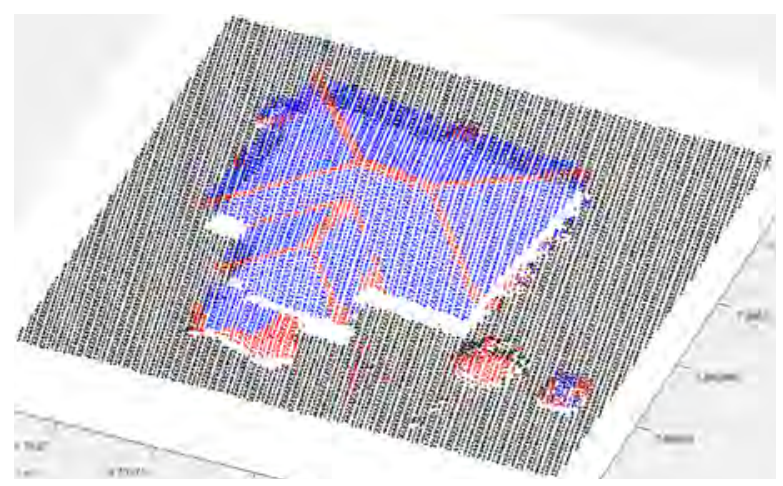

Fig. 3. ALS point clouds segmentation - Ground points (black), non-ground points (blue), and feature points (red)

For the non-ground point clouds, a neighbourhood connection is established using Delaunay triangulation (see Figure 4a). The key idea behind the candidate region identification is to remove the triangles connecting the edges between different objects. Accordingly, the edges of any triangle having a length larger than twice of the point cloud spacing are classified anomalous and thus removed as shown in Figure 4(b). Then, following the new boundary approximation algorithm (not presented here), the candidate building regions are identified and their corresponding boundaries are estimated.

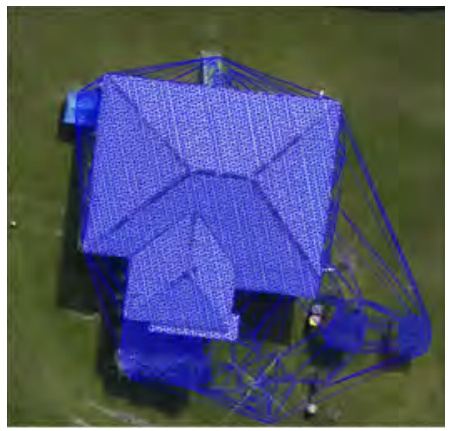

(a)

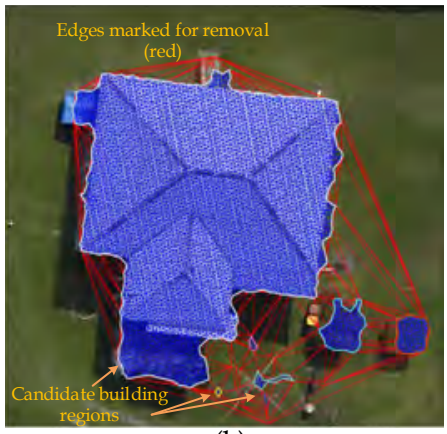

(b)
Fig. 4. Candidate Building regions identification (overlaid on input image for demonstration) - (a) Triangularisation (blue), and (b) the identified candidate regions (red - edges to remove)

\subsection{Roof feature extraction}

The proposed segmentation method to extract the coplanar primitives is entirely based on the region-growing algorithm. Since, all ALS data sets have inherent noise and surface roughness, therefore, we choose only the critical parameters for the region growing, such as surface flatness [12] and normal orientation [14] as a coherence criteria while a flat plane tolerance and plane fitting error [15] to distinguish the superpose surfaces. The candidate building regions are processed iteratively but the ones having anisotropic points larger than $95 \%$ are removed considering as trees (collection of several anisotropic surfaces). The segmentation process begins with the selection of a seed point. A point that does not belong to sharp features and has the most flatness value is identified as a seed believing that the region growing will be more successful for areas where the spatial variation is the least.

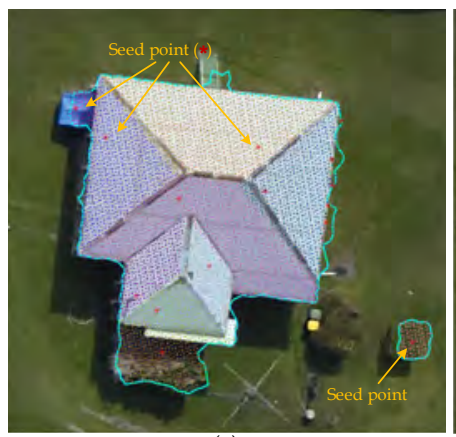

(a)

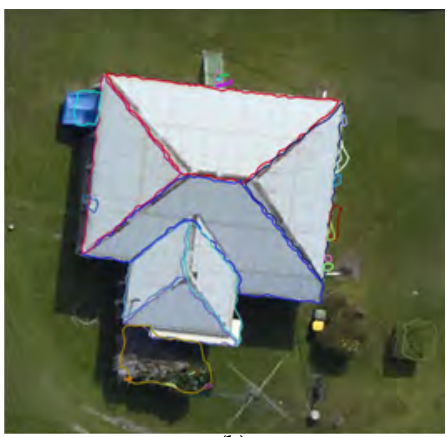

(b)
Fig. 5. Roof plane extraction outcome sketched on the test image - (a) Segmented ALS data, and (b) Estimated planes boundaries

After getting a seed point, we take its $k$ local neighbourhood points $N_{p}$. To maintain the topology of the roof primitives, the points which have the angular difference between the normal vector of seed and $N_{P}$ within a predefined threshold $\theta_{t}$, a fictitious plane is fitted using the least squares method. If the plane fitting error and the difference between point clouds' height and fitted plane's height are smaller than their fitting error $\varepsilon_{t}$ and flat plane tolerance $\xi_{t}$ respectively, these points are added into the region, which is grown until new neighbouring points meet the above criteria. Otherwise, a new seed point is chosen and repeat 
the process until all the point clouds are assigned to a plane. The segmentation outcome of the test data set is sketched in Figure 5. For all the experiments in this study, the proposed segmentation method adopts the threshold values for $\theta_{t}=10^{0}$ from Chen et al. [14] and $\varepsilon_{t}=0.1 \mathrm{~m}$ and $\xi_{t}=0.15 \mathrm{~m}$ from Awrangjeb and Fraser [15] for brevity.

\subsection{Plane refinement and boundary approximation}

As a result of the segmentation, some of the extracted planes may belong to trees and other non-building structures. We call these the false positive planes, which can be removed using a rule-based procedure following the criteria in [15]. The planar features, such as its area, standard deviation, local neighbourhood, and the presence of straight line segments around its boundary, are used to decide whether a plane is a false positive.

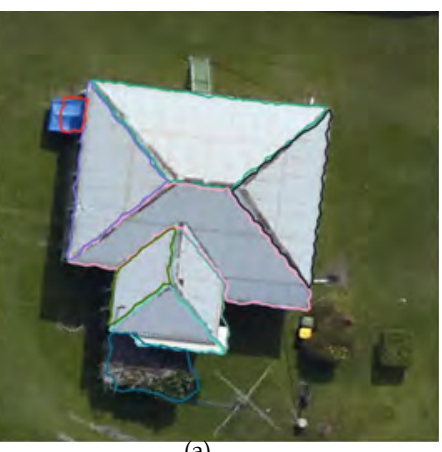

(a)

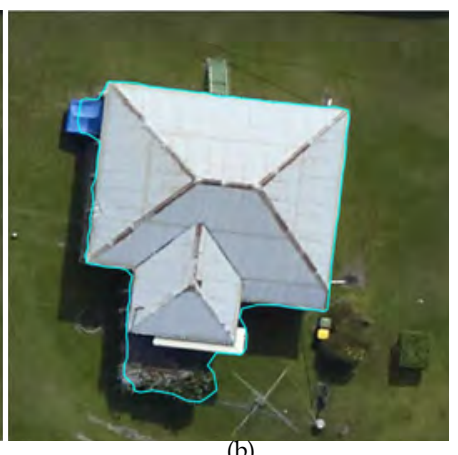

(b)
Fig. 6. (a) Extracted planes boundaries, and (b) Extracted building boundary. Both are laid on the test image for a demo

It is observed that a plane fitted on a tree is usually small in size and has a higher standard deviation value than a plane on a building. Therefore, a plane is discarded as a false positive regardless of the other parameters either if its area is $\leq$ $1 \mathrm{~m}^{2}$ or the standard deviation of point clouds in a given plane is higher than the user defined threshold. Moreover, the presence of long straight lines $(\geq 3 \mathrm{~m}$ ) along the boundary of a plane, is also utilised to remove the false planes on trees and other nonbuilding structures [15]. In addition, the remaining planes if exist in the local neighbourhood of a false positive plane are also removed as false alarms. Figure 6(a) shows the final extracted roof planes after the refinement process. Each candidate building region is further processed iteratively to identify the individual buildings. Therefore, point clouds of the neighbouring roof planes are combined and a boundary estimation algorithm (described earlier) is used to estimate the boundary of the buildings present in a given candidate region. Figure 6(b) shows the building boundary for the test scene.

\section{PERFORMANCE EVALUATION}

The proposed method is demonstrated and evaluated using two real benchmark data sets, ISPRS (Vaihingen Germany, Area 1 - VA 1) and Australian (Hervey Bay - HB). The VA 1 is situated in the centre of the city and has a LiDAR density of 3.5 points $/ \mathrm{m}^{2}$. This area is characterised by dense construction consisting of historic buildings having complex shapes. The HB data set has a LiDAR density of 12 points $/ \mathrm{m}^{2}$. Topographically, the area is flat containing residential buildings with dif- ferent level of vegetation. The objective evaluation in this study employs the threshold-based system [16] for the ISPRS data set and automatic and threshold-free evaluation system [17] for the Australian data set because of unavailability of a uniform evaluation system [16].

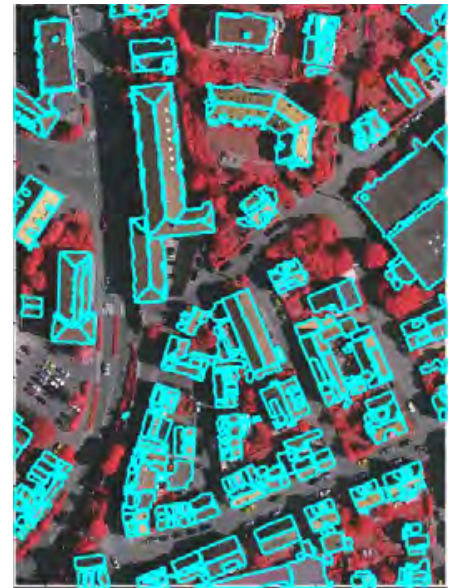

(a)

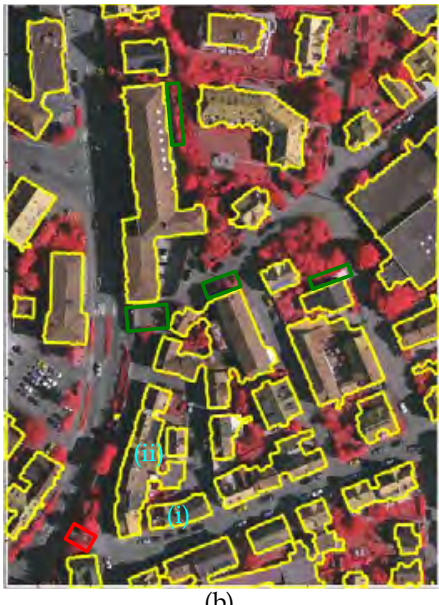

(b)
Fig. 7. (a) Extracted planes boundaries, and (b) Extracted building boundaries. Both are laid on the Area 1 image for a demo

Quantitative and qualitative results of the building extraction are presented in Table 1 and Figures 7 and 8 respectively. Table 1 shows that the proposed method extracted all the buildings from VA 1 and $\mathrm{HB}$ data sets with an object-based completeness of $89.2 \%$ and $100 \%$ respectively with a correctness above $97 \%$. Similarly, $100 \%$ per-object completeness and correctness were achieved on the buildings larger than $50 \mathrm{~m}^{2}$. Besides, $88.3 \%$ and $95.20 \%$ per-pixel completeness was achieved with a respective correctness of $89.8 \%$ and $92.21 \%$ for both VA 1 and $\mathrm{HB}$ data sets. But, the buildings and few attachments marked with red and green rectangles (see Figure $7 \mathrm{~b}$ ) were not identified because of their height less than the height threshold. Few under-segmentation issues were observed in Figures 7(b) (Labels $\mathrm{i}$ and ii) and 8(b) (Label iii) when two very close buildings were merged due to a narrow gap equal to the ALS data density.

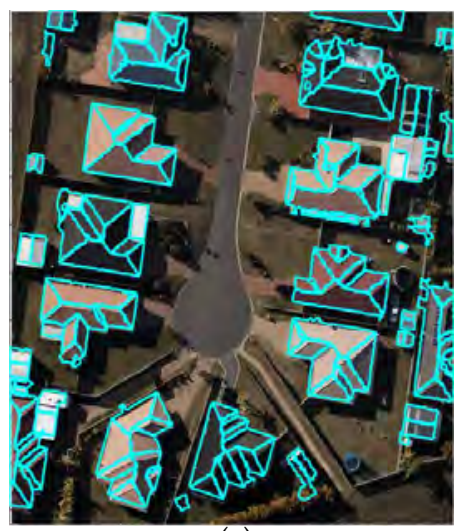

(a)

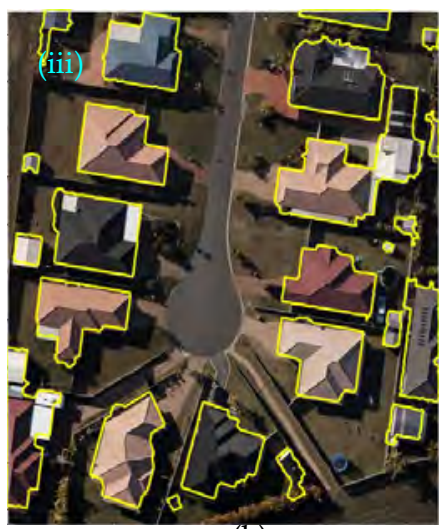

(b)
Fig. 8. (a) Extracted planes boundaries, and (b) Extracted building boundaries. Both are laid on the Hervey Bay image for demo

Compared with two automatic and data-driven methods, Mon2 [15] and Yang [18], the proposed method extracted buildings with a better per-object and pixel accuracy. To the best of 


\begin{tabular}{|c|c|c|c|c|c|c|c|c|c|c|c|}
\hline Methods & Data set & $C_{m}$ & $C_{r}$ & $Q_{l}$ & $C_{m, 50}$ & $C_{r, 50}$ & $\mathrm{M} / \mathrm{N} / \mathrm{B}$ & $C_{m p}$ & $C_{r p}$ & $Q_{l p}$ & $R M S E$ \\
\hline \multirow{2}{*}{ Proposed } & VA 1 & 89.2 & 97.1 & 86.8 & 100 & 100 & $0 / 7 / 0$ & 88.3 & 89.8 & 80.3 & 0.95 \\
\hline & HB & 100 & 100 & 100 & 100 & 100 & $0 / 1 / 0$ & 95.20 & 92.21 & 88.12 & 0.54 \\
\hline \multicolumn{12}{|c|}{ Evaluation results of the comparative methods } \\
\hline \multirow{2}{*}{ Mon2 } & VA 1 & 89.2 & 91.4 & 82.3 & 100 & 100 & $0 / 6 / 0$ & 88.1 & 90.0 & 80.3 & 1.0 \\
\hline & $\mathrm{HB}$ & 86.4 & 100 & 86.4 & 100 & 100 & $0 / 1 / 0$ & 91.7 & 95.6 & 87.9 & 1.42 \\
\hline Yang & VA 1 & 81.1 & 96.8 & - & 100.0 & 96.6 & $-/-/-$ & 87.9 & 91.2 & - & 0.9 \\
\hline
\end{tabular}

Table 1. Building detection evaluation results of the proposed and comparative methods using Vaihingen (VA 1) and Hervey Bay (HB) data sets. Object-based $C_{m}=$ completeness, $C_{r}=$ correctness, $Q_{l}=$ quality, $\left(C_{m, 50}\right.$ and $C_{r, 50}$ are for buildings over $\left.50 \mathrm{~m}^{2}\right) . \mathrm{M}$ $=$ over-segmentation, $\mathrm{N}=$ under-segmentation, $\mathrm{B}=$ both under- and over-segmentation. Pixel-based $C_{m p}=\operatorname{completeness}$ and $C_{r p}=$ correctness, and $Q_{l p}=$ quality are in percentage. $\mathrm{RMSE}=$ planimetric accuracy in metres. - means results unavailable

our knowledge, Mon2 is the only method evaluated on HB data set using the evaluation system in [17]. Table 1 shows a constantly higher correctness of the proposed method as compared to the counterparts which demonstrate its robustness. Moreover, a better planimetric accuracy (RMSE) of the extracted outline was achieved which is around two times the horizontal point spacing of the ALS data.

\section{CONCLUSION}

An automatic and robust data-driven method is proposed for planar surfaces and building extraction from laser scanning data. It combines PCA and LRSCPK to estimate the saliency features while preserving the anisotropic surfaces and make the segmentation more resistive towards noises. Results for the experiments on real data sets show that the proposed method produces accurate results at a high per-object and area accuracies. The experimental results demonstrated that our method is completely free from many-to-many and over-segmentation errors, which is imperative for a robust approach. Future work aims to regularise the building outlines and develop the 3D city models using the planar surfaces.

\section{References}

[1] SAN Gilani, M. Awrangjeb, and G. Lu, "Fusion of lidar data and multispectral imagery for effective building detection based on graph and connected component analysis," Int. Arch. Photogramm. Remote Sens. Spat. Inf. Sci., vol. I, pp. $65-72,2015$.

[2] J. Zhang, J. Cao, X. Liu, J. Wang, J. Liu, and X. Shi, "Point cloud normal estimation via low-rank subspace clustering," Comput. Graph., vol. 37, no. 6, pp. 697-706, 2013.

[3] A. Boulch and R. Marlet, "Fast and robust normal estimation for point clouds with sharp features," in Comput. Graph. Forum, 2012, vol. 31, pp. 1765-1774.

[4] H. Hoppe, T. DeRose, T. Duchamp, J. McDonald, and W. Stuetzle, "Surface reconstruction from unorganized points," SIGGRAPH Comput. Graph., vol. 26, no. 2, pp. 71-78, 1992.

[5] N. J. Mitra, A. Nguyen, and L. Guibas, "Estimating surface normals in noisy point cloud data," Int. J. Comput. Geom. Appl., vol. 14, no. 04n05, pp. 261-276, 2004.

[6] S. Fleishman, D. Cohen-Or, and C. Silva, "Robust moving least-squares fitting with sharp features," in ACM Trans. Graph., 2005, vol. 24, pp. 544-552.
[7] D. Chen, L. Zhang, J. Li, and R. Liu, "Urban building roof segmentation from airborne lidar point clouds," Int. J. Remote Sens., vol. 33, no. 20, pp. 6497-6515, 2012.

[8] P. Luo, Z. Wu, C. Xia, L. Feng, and B. Jia, "Robust normal estimation of point cloud with sharp features via subspace clustering," in Fifth Int. Conf. on Graph. Image Process, 2014, pp. 90691S-90691S.

[9] G. Guennebaud and M. Gross, "Algebraic point set surfaces," in ACM Trans. Graph., 2007, vol. 26, p. 23.

[10] B. Li, R. Schnabel, R. Klein, Z. Cheng, G. Dang, and S. Jin, "Robust normal estimation for point clouds with sharp features," Comput. Graph., vol. 34, no. 2, pp. 94-106, 2010.

[11] R. Hoffman and A. Jain, "Segmentation and classification of range images," IEEE Trans. Pattern Anal. Mach. Intell., , no. 5, pp. 608-620, 1987.

[12] L. Zhu, M. Lehtomäki, J. Hyyppä, E. Puttonen, A. Krooks, and H. Hyyppä, "Automated 3d scene reconstruction from open geospatial data sources: Airborne laser scanning and a 2d topographic database," Remote Sens., vol. 7, no. 6, pp. 6710-6740, 2015.

[13] M. Pauly, R. Keiser, and M. Gross, "Multi-scale feature extraction on point-sampled surfaces," in Comput. Graph. Forum, 2003, vol. 22, pp. 281-289.

[14] D. Chen, L. Zhang, PT Mathiopoulos, and X. Huang, "A methodology for automated segmentation and reconstruction of urban 3-d buildings from als point clouds," IEEE J. Sel. Top. Appl. Earth Obs. Remote Sens., vol. 7, no. 10, pp. 4199-4217, 2014.

[15] M. Awrangjeb, G. Lu, and C. S. Fraser", "Automatic building extraction from lidar data covering complex urban scenes," Int. Arch. Photogramm. Remote Sens. Spat. Inf. Sci., vol. I, pp. 25-32, 2014.

[16] M. Rutzinger, F. Rottensteiner, and N. Pfeifer, "A comparison of evaluation techniques for building extraction from airborne laser scanning," IEEE J. Sel. Top. Appl. Earth Obs. Remote Sens., vol. 2, no. 1, pp. 11-20, 2009.

[17] M. Awrangjeb and C.S. Fraser, "An automatic and threshold-free performance evaluation system for building extraction techniques from airborne lidar data," IEEE $J$. Sel. Top. Appl. Earth Obs. Remote Sens., vol. 7, no. 10, pp. 4184-4198, Oct 2014.

[18] Y. Bisheng, X. Wenxue, and D. Zhen, "Automated extraction of building outlines from airborne laser scanning point clouds," IEEE Geosci. Remote Sens. Lett., vol. 10, no. 6, pp. 1399 - 1403, Nov 2013. 